\title{
Facteurs de risque de chutes chez les aînés vivant dans la communauté et ayant recours aux services de sou- tien à domicile : modèle de risques élargi avec cova- riables dépendantes du temps et événements récurrents
}

BS Leclerc, M.Sc. (1); C Bégin, M.Sc. (1); É Cadieux, M.Sc. (1); L Goulet, M.D., Ph.D. (2); N Leduc, Ph.D. (2);

M-J Kergoat, M.D. (3); P Lebel, M.D. (3,4)

\section{Résumé}

Dans les études longitudinales, il devient difficile de déterminer les facteurs de risque de chutes, car l'exposition varie durant la période de suivi et les sujets peuvent subir un événement plus d'une fois. Or, les analystes font peu de cas de ces considérations et n'utilisent pas nécessairement les bonnes méthodes statistiques. En règle générale, on se concentre sur la proportion des personnes ayant fait une chute ou le temps écoulé jusqu'à la première chute. Dans un cas comme dans l'autre, on fait abstraction de l'hypothèse fondamentale de l'indépendance des événements et on écarte par le fait même des données pertinentes. Dans le présent article, nous examinons les méthodes en usage actuellement et nous proposons une extension du modèle de risques de Cox. Nous illustrons cette version étendue du modèle par une étude des facteurs de risque susceptibles d'être associés à des chutes parmi un groupe de 959 personnes âgées. Enfin, nous comparons les résultats obtenus avec la méthode de Wei, Lin et Weissfeld (ci-après désignés WLW) et ceux obtenus avec plusieurs autres méthodes. Les facteurs d'exposition étudiés qu'ils soient fixes ou variables dans le temps comprennent les caractéristiques socio-démographiques, l'indice de masse corporelle (IMC), le risque nutritionnel, la consommation d'alcool, les dangers de l'environnement domiciliaire, la démarche et l'équilibre, et la consommation de médicaments. Notre étude révèle que les méthodes utilisées couramment pour analyser les facteurs de risque de chutes sont inadéquates, car elles créent un biais appréciable comparativement au modèle de WLW, qui utilise des covariables dépendantes du temps. Les résultats de notre étude montrent également qu'il peut être vain de modéliser la première survenue d'un événement, étant donné que le risque de survenue varie entre les chutes.

Mots clés : Chutes accidentelles, modèle de Cox, aînés, risques environnementaux, distribution binomiale négative, modèle de risques, analyse de régression, analyse de survie, modèles logistiques

\section{Introduction}

Les chutes représentent un problème fréquent et récurrent qui a des conséquences graves pour les personnes âgées et le système de soins de santé1. On détermine habituellement les facteurs de risque de chutes par des études d'observation prospectives. Or, ces études présentent parfois des lacunes semblables à celles que l'on trouve dans plusieurs autres types d'études, p. ex., suivi impossible ou durée variable des suivis. La détermination des facteurs de risque de chutes est compliquée en outre par le fait que l'exposition varie au cours du suivi et qu'un même individu peut subir un événement plus d'une fois. Les analystes font peu de cas de ces considérations et les méthodes statistiques qu'ils utilisent sont par conséquent inefficaces, ce qui peut avoir faussé les estimations relatives à certains prédicteurs ou produit des résultats ambigus. Par surcroît, cette négligence peut avoir contribué à éluder des questions d'un grand intérêt sur le plan clinique ${ }^{2-5}$.

Il y a plus de 15 ans, Cumming, Kelsey et Nevitt $^{6}$ émettaient l'avis qu'il fallait accorder plus d'attention aux mesures répétées des facteurs de risque et au taux d'incidence des chutes. Malgré cela, peu de chercheurs ont remis en question leur façon de faire et leur méthode d'analyse des données. Le commentaire des auteurs aura plutôt provoqué la réaction inverse : les chercheurs ont contourné les difficultés méthodologiques en écartant une grande quantité de renseignements utiles.

Le présent article a pour objet de sensibiliser davantage les chercheurs à un certain nombre de considérations épidémiologiques et statistiques. Nous allons examiner les fondements statistiques des méthodes utilisées dans les études sur les chutes, exposer les notions de covariables dépendantes du temps et de récurrence des événements, et analyser les méthodes statistiques courantes qui ont recours à ces notions. Nous proposons en

Coordonnées des auteurs

1 Direction de santé publique et d'évaluation, Agence de la santé et des services sociaux de Lanaudière, Joliette (Québec)

2 Groupe de recherche interdisciplinaire en santé, Université de Montréal, Montréal (Québec)

3 Centre de recherche, Institut universitaire de gériatrie de Montréal, Montréal (Québec)

4 Centre d'expertise sur la santé des personnes âgées et des aidants, Institut universitaire de gériatrie de Montréal, Montréal (Québec)

Correspondance : Bernard-Simon Leclerc, Service de surveillance, recherche et évaluation, Direction de santé publique et d'évaluation, Agence de la santé et des services sociaux de Lanaudière, 245, rue du Curé-Majeau, Joliette (Québec) J6E 8S8, Tél. : 450-759-1157, poste 4324, Courriel : Bernard-Simon_Leclerc@ssss. gouv.qc.ca 
outre une extension du modèle des risques proportionnels de Cox, que nous illustrons par une étude des facteurs de risque susceptibles d'être associés à des chutes chez un groupe de personnes âgées qui vivent à domicile. Enfin, nous comparons les résultats produits par les différentes méthodes statistiques.

\section{Fondements statistiques des méthodes utilisées dans les études sur les chutes}

On se sert de différentes méthodes pour étudier les facteurs de risque de chutes récurrentes. Leur analyse est compliquée par la corrélation intra-sujet. En d'autres mots, la survenue d'un événement influe sur le risque de survenue du prochain événement. Le fait de ne pas tenir compte de cette corrélation dans les données résulte en une sous-estimation de la variance ordinaire, ce qui se traduit par des intervalles de confiance trop étroits et des tests de signification favorisant outre mesure le rejet de l'hypothèse nulle ${ }^{3,5,7}$.

La figure 1 présente un résumé de quelques méthodes analysées dans le présent article. Une méthode élémentaire pour l'étude du phénomène des chutes chez les personnes âgées consiste à indiquer tout simplement la proportion des personnes ayant fait une chute (les sujets qui sont tombés au moins une fois durant une période définie arbitrairement) ou le temps écoulé jusqu'à la première chute ${ }^{8}$. Dans un cas comme dans l'autre, on fait l'économie de l'hypothèse fondamentale de l'indépendance des événements. Il n'empêche que l'on pourrait utiliser plus efficacement toutes les données disponibles sur chaque individu ${ }^{4,8,9}$. Les auteurs d'un article de premier plan affirment que l'étude du taux d'incidence des chutes doit être une priorité en matière de santé publique ${ }^{6}$, notamment en ce qui concerne les personnes âgées plus frêles ${ }^{10}$. Le problème qui se pose lorsqu'on veut analyser toutes les chutes est que certaines personnes sont plus prédisposées aux chutes que d'autres; elles courent donc plus de risque de se blesser en tombant que les personnes qui feront une seule chute. Par ailleurs, le choix de la variable étudiée selon que l'accent est mis sur les personnes ayant fait une chute ou le taux d'incidence des chutes peut influer sur la conclusion, c'est-à-dire à savoir si un facteur d'exposition particulier constitue un facteur de risque. Les facteurs d'exposition fixes sont plus susceptibles d'être associés à la condition de " personne ayant fait une chute " que les facteurs d'exposition variables dans le temps ${ }^{6}$.

D’autres méthodes ont été proposées pour résoudre la question des événements récurrents. Parmi celles-là notons la régression binomiale négative, des extensions du modèle des risques proportionnels de Cox et une version modifiée de la régression logistique. Dans le premier cas, la variable dépendante est le nombre d'événements, pour un individu, ajusté en fonction de la période de suivi, c'est-à-dire le nombre de chutes subies par une personne, divisé par la durée du suivi (Figure 1) ${ }^{4,11}$. Comme la distribution binomiale négative compte un paramètre de plus que la distribution de Poisson, elle se concilie naturellement avec la surdispersion (c'est-à-dire le phénomène selon lequel la variance est supérieure à la moyenne) ${ }^{8}$. Cette méthode est donc robuste pour les données qui présentent des rapports de dépendance et appropriée pour les événements récurrents et fréquents.

Une des difficultés qui se pose avec l'utilisation du nombre d'événements est que l'on doit supposer invariable dans le temps la probabilité de survenue d'un événement pour un participant. Afin d'illustrer de façon éloquente cette difficulté, considérons un nombre d'événements identique chez trois participants, qui sont suivis sur une période de trois ans et ont fait chacun trois chutes. Le premier participant est tombé une fois chaque année, le deuxième, trois fois dans la première année et le troisième, trois fois dans la dernière année. La variable étudiée fait abstraction du moment où surviennent les événements ${ }^{8}$. Par conséquent, la modélisation du nombre d'événements par la régression binomiale négative n'est peut-être pas la méthode la plus appropriée lorsque la valeur de covariables importantes ou la probabilité de survenue d'un événement varie dans le temps ${ }^{3}$. Il est plus efficace et plus juste de modéliser la durée des intervalles entre les événements à l'aide des méthodes d'analyse du temps écoulé jusqu'à un événement ${ }^{9}$. Au lieu de se centrer sur le nombre de cas, les méthodes de ce type considèrent le temps écoulé entre les chutes. Si le taux d'incidence est élevé, les intervalles entre les événements seront courts, et vice-versa ${ }^{3}$.

Par ailleurs, les facteurs de risque mesurés dont nous cherchons à évaluer les effets sont ordinairement des variables fixées, qui ont été définies au moment de l'examen initial' ${ }^{2}$. Ces facteurs représentent les caractères intrinsèques du sujet (p. ex., le sexe), les expositions aux risques antérieures (p. ex., les chutes antérieures) ou les risques présents au départ (p. ex., l'usage de médicaments). On ne tient pas compte des risques qui peuvent surgir en cours de route ou qui varient dans le temps. Parmi les facteurs d'exposition précédant de peu une chute et qui peuvent être à l'origine de celleci notons les dangers de l'environnement domiciliaire et la consommation d'alcool et de médicaments. Un avantage notable de la méthode d'analyse du temps écoulé jusqu'à un événement est sa capacité d'intégrer les covariables dépendantes du temps ${ }^{3}$.

Les modèles de risques comprennent le processus de comptage de Andersen et Gill $^{16}$ (ci-après désignés AG), le modèle conditionnel de Prentice, Williams et Peterson $^{17}$ (PWP) et le modèle marginal de Wei, Lin et Weissfeld ${ }^{18}$ (WLW). Aucune de ces méthodes ne modélise explicitement la structure de dépendance entre les temps de survie. On recourt plutôt à des estimations robustes de la variance pour tenir compte de la corrélation des observations chez un même sujet; c'est ce que nous appelons les modèles de risques corrigés par un estimateur robuste de la variance ${ }^{12-15}$.

On peut distinguer les modèles de risques en fonction de qui est inclus dans l'ensemble des personnes " exposées au risque » à chaque épisode ${ }^{15,19}$. L'approche de AG repose sur l'hypothèse stricte selon laquelle le risque d'un événement chez un sujet donné n'est aucunement déterminé par les événements antérieurs, à moins 
que l'on intègre dans le processus, en qualité de covariable dépendante du temps, un terme qui rende compte de cette influence (c.-à-d. le nombre de chutes antérieures $)^{3,7}$. Autrement dit, les données sur un sujet ayant subi plus d'un événement peuvent être présentées comme les données relatives à plusieurs sujets, où chacun de ces sujets entre dans l'analyse avec un décalage et fait l'objet d'un suivi jusqu'au prochain événement (Figure 1). Ce modèle fait abstraction de l'ordre des événements, c'est-à-dire qu'on ne peut différencier les chutes entre elles, laissant ainsi chaque sujet "exposé » au risque d'un événement, pourvu que ce sujet fasse encore l'objet d'un suivi au moment où survient l'événement $t^{3,7,8,13-15,19}$.

Le modèle de PWP repose sur l'idée qu'un sujet n'est pas techniquement exposé au risqued'unévénement ultérieur tant et aussi longtemps qu'il n'est pas reconnu comme ayant subi tous les événements antérieurs. Pour ce faire, on stratifie les données selon l'ordre des événements. En conséquence, l'ensemble des personnes exposées au risque au moment $t$ pour le $k^{\mathrm{e}}$ événement se compose uniquement des sujets faisant l'objet de l'étude au moment $t$ qui ont déjà subi $k$-1 événements (non illustré dans la Figure 1$)^{13-15,19}$. Or, Robertson ${ }^{20}$ affirme que l'hypothèse conditionnelle de l'ordre des événements n'est pas valable en ce qui concerne les chutes de personnes âgées. Afin d'illustrer les propos de cet auteur (recueillis dans une communication personnelle), supposons qu'une personne est tombée dans sa cuisine après avoir mis le pied dans une flaque d'eau, mais sans se blesser, et que par la suite elle est tombée sur le trottoir à l'extérieur et s'est fracturée la hanche. Cette personne est réputée exposée au risque de chute dès le début de la période de suivi, c'est-à-dire qu'il est faux d'affirmer que la période d'exposition au risque pour ce qui est de la seconde chute ne débute qu'après la survenue de la première chute.

Selon le modèle marginal de WLW, l'ensemble des personnes exposées au risque comprend tous les patients faisant l'objet de l'étude qui n'ont pas encore subi le $k^{\mathrm{e}}$ événement. La période d'exposition au risque pour chaque événement débute

\section{FIGURE 1}

\section{Représentation schématique des modèles statistiques utilisés pour l'étude des facteurs de risque de chutes}

(inspirée d'une figure parue dans Robertson, Campbell et Herbison ${ }^{8}$ )

Sujet hypothétique faisant l'objet d'un suivi sur 12 jours et subissant une chute au $5^{\mathrm{e}}$ et au $8^{\mathrm{e}}$ jour. Supposons que (0) désigne l'absence de chute et (1), la survenue d'une chute; $x_{i}$ représente un facteur de risque pour le sujet $i$, mesuré à la période $t$, et $k_{i}$, le nombre de chutes. Donc, le risque de base est représenté par $\lambda_{0}(t)$, le risque de chute pour le sujet $i$,

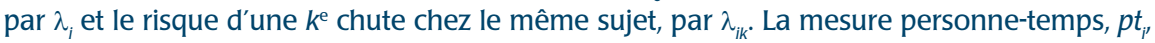
est la durée de l'exposition au risque pour le sujet $i, \beta^{\prime} x$, la taille de l'effet du facteur $x$, et $p$, la probabilité de survenue d'un événement chez les sujets exposés, $e$, et les sujets non exposés, $u$.

Régression de Cox ordinaire. Un enregistrement unique couvre la période allant de l'entrée dans le projet jusqu'au moment de la première chute et exclut toute information subséquente. Pour les personnes qui n'ont fait aucune chute, on impute la durée totale du suivi. La variable dépendante est le temps écoulé jusqu'à la première chute.

$\vdash \perp \perp \perp \perp_{(1)}$

$$
\log \left(\frac{\lambda_{i}(t)}{\lambda_{0}(t)}\right)=\beta^{\prime} x_{i}(t)
$$

Régression de Cox de Andersen-Gill. Trois enregistrements couvrent la période allant de l'entrée dans le projet jusqu'à la première chute, celle allant de la première chute à la seconde chute, et celle allant de la dernière chute à la fin du suivi, cette dernière période ne comportant aucune chute. La variable dépendante est le temps écoulé jusqu'à chaque chute.

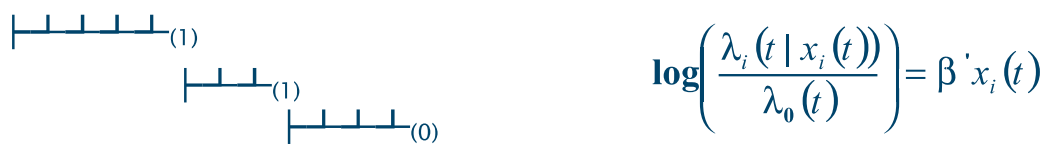

Régression marginale de Wei, Lin et Weissfeld. Trois enregistrements. Chaque période durant laquelle survient une chute, ainsi que la dernière période, où il y a absence de chute, constitue une strate indépendante et la durée totale est mesurée à partir de l'entrée dans le projet. La variable dépendante est le temps écoulé jusqu'à chaque chute.

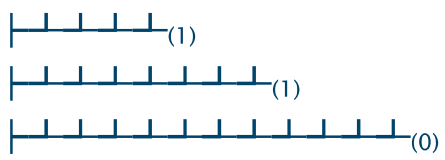

$$
\log \left(\frac{\lambda_{i k}\left(t \mid x_{i k}(t)\right)}{\lambda_{0 k}(t)}\right)=\beta_{k} x_{i k}(t)
$$

Régression binomiale négative. Un enregistrement unique couvre la période allant de l'entrée dans le projet jusqu'à la fin du suivi et indique simplement le nombre total de chutes et la durée du suivi pour chaque sujet. La variable dépendante est le nombre de chutes.

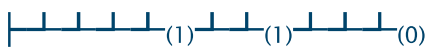

$$
\log \left(\frac{k_{i}}{p t_{i}}\right)=\beta_{0}+\beta^{\prime} x_{i}
$$

Régression logistique. Un enregistrement unique, qui fait abstraction de la durée du suivi et des chutes récurrentes pour chaque sujet. La variable dépendante binaire est la condition de personne ayant fait une chute

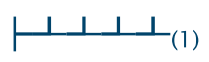


en même temps que la période de suivi pour chaque sujet. En outre, chaque sujet est réputé exposé au risque pour tous les événements, peu importe le nombre d'événements qu'il a réellement subis. Le modèle de WLW n'impose pas de structure de dépendance entre les temps de survie qui sont liés. Il fait donc abstraction de l'ordre des événements, mais tient compte des événements antérieurs en classant chaque épisode dans une strate

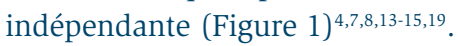

L'analyse de régression logistique est la méthode le plus couramment utilisée en recherche épidémiologique. D’Agostino et coll. ${ }^{21}$ montrent que la régression logistique groupée est semblable à une régression de Cox avec covariables dépendantes du temps. C'est ce qui rend la méthode intéressante lorsqu'il s'agit d'évaluer le rapport entre les facteurs de risque et le développement d'une maladie. O'Loughlin ${ }^{22}$ a eu recours à cette méthode dans une étude sur les chutes. L'utilisation de cette variante de la régression logistique est bien fondée théoriquement lorsque les intervalles entre les évaluations des facteurs de risque sont courts, la probabilité de survenue d'un événement dans un intervalle est faible, et l'ordonnée à l'origine du modèle de régression logistique groupée est la même pour tous les intervalles ${ }^{21}$. Les critères statistiques et la structure des données définis pour la régression logistique groupée sont très comparables à ceux établis pour le modèle de AG. On pose comme hypothèse que chacune des périodes de suivi d'un sujet unique représente les périodes de suivi de plusieurs sujets. La méthode regroupe les sujets exposés au risque et les événements qui se sont produits à chaque période. Pour tester cette hypothèse, on définit comme variable catégorielle le numéro de l'entrevue de suivi. De même, on tient compte du lien de dépendance entre les chutes chez une même personne en considérant la survenue de chutes antérieures comme une variable explicative ${ }^{23}$.

Toutefois, la variable étudiée, ainsi que la structure des observations des périodes diffèrent d'une méthode à l'autre. En effet, le modèle de AG définit les intervalles de temps en fonction de la date précise des événements. Par exemple, le premier intervalle est la période qui s'étend de la date d'entrée dans l'échantillon observé jusqu'à la survenue de l'événement; le deuxième intervalle est la période qui s'étend entre le premier événement et l'événement suivant, et ainsi de suite (Figure 1) ${ }^{15}$. A contrario, la régression logistique utilise des dates fixes établies par le chercheur. Par exemple, on effectue un contrôle chaque mois à la même date pour faire une mise à jour des facteurs de risque et recueillir de l'information sur les chutes survenues au cours de la période d'observation (non illustré dans la Figure 1) 22,23. L'analyse ci-dessus est fondamentalement une étude des personnes ayant fait une chute, par opposition à celles qui n'en ont pas fait, sur de courtes périodes successives ${ }^{22}$. Même si, dans l'ensemble, l'analyse prévoit la possibilité qu'un sujet subisse plus d'un événement, elle laisse de côté toutes les chutes additionnelles qui peuvent survenir dans un même intervalle. Il semble évident que si l'on considère que, pour une même période (un mois en l'occurrence), trois chutes sont l'équivalent d'une seule, on "sacrifie » l'information qui traduit l'intensité des phénomènes à court terme.

Le choix de l'un ou l'autre de ces modèles doit être fondé sur des a priori concernant le type de rapports qui existent entre les covariables et le risque de chutes. La régression binomiale négative, la régression de AG et la régression logistique groupée n'établissent pas une distinction entre les divers événements qui se succèdent, ce qui limite le risque de base; en outre, les coefficients de régression ne varient pas selon l'ordre de récurrence. Il est largement reconnu que l'existence de chutes antérieures est un prédicteur des chutes à venir ${ }^{10,23}$. Intuitivement, nous nous attendrions à ce que la cause d'une première chute diffère de celles des chutes qui vont suivre. Les prédicteurs de la chute qui survient accidentellement peuvent être différents de ceux des chutes récurrentes liées à la condition physique de la personne $e^{24,25}$. D'où le fait que les chercheurs et les praticiens voudraient connaître non seulement l'incidenceglobale des covariables sur le risque d'épisodes multiples confondus, mais aussi l'incidence de chacune des variables indépendantes sur le risque de survenue d'un premier événement, d’un deuxième événement, etc. Contrairement à la méthode de WLW, la régression binomiale, la régression de AG et la régression logistique groupée ne nous éclairent pas sur ces questions. Compte tenu de la structure des données à analyser et de la question étudiée, la méthode de WLW devrait en principe mieux se prêter à l'étude des facteurs de risque de chutes.

\section{Méthodes}

\section{Sujets et procédures}

Le recrutement des sujets volontaires a été effectué entre mars 2002 et juillet 2005, afin de constituer une cohorte ouverte comprenant des personnes de 65 ans ou plus qui vivent dans la communauté et qui reçoivent des services de soins ou de soutien à domicile. Étaient exclues les personnes qui ne parlaient ni français ni anglais, celles qui ne pouvaient franchir une distance de plus de six mètres à pied, et celles qui souffraient d'une diminution des facultés de communication et des facultés cognitives. Tous les sujets ont donné leur consentement en connaissance de cause. L'étude a reçu l'aval des autorités de chaque centre participant.

La présente étude s'inscrit dans le cadre d'un projet de recherche visant à évaluer l'utilité d'un programme d'interventions préventives interdisciplinaires ${ }^{26}$. Un thérapeute qualifié de la réadaptation physique se rendait au domicile des participants au début de la période d'observation, puis aux six mois, afin de procéder à une réévaluation du risque de chutes. On définissait la chute comme un événement par suite duquel le sujet se retrouve involontairement au sol, au plancher ou sur d'autres surfaces plus basses (p. ex. l'escalier). Étaient exclues les chutes liées à la pratique d'un sport ${ }^{23}$. 
TABLEAU 1

Estimations du risque relatif ajusté des facteurs de chute chez les aînés vivant dans la communauté, selon différentes méthodes de régression statistique

\begin{tabular}{|c|c|c|c|c|c|c|c|c|}
\hline \multirow{2}{*}{$\begin{array}{l}\text { Facteur de risque } \\
\text { Dangers de l'eviron. domicil. } \\
\text { (nombre) }\end{array}$} & $\begin{array}{c}1 \\
\text { Logistique } \\
\text { (personnes } \\
\text { ayant fait } \\
\text { une chute) }\end{array}$ & $\begin{array}{c}2 \\
\begin{array}{c}2 \\
\text { Binomiale } \\
\text { négative }\end{array} \\
\text { (toutes } \\
\text { chutes) }\end{array}$ & $\begin{array}{c}\text { 3a } \\
\text { Standard } \\
\text { Cox/WLW } \\
\text { (première } \\
\text { chute) }\end{array}$ & $\begin{array}{c}4 a \\
\text { AG Cox } \\
\end{array}$ & $\begin{array}{c}5 \mathbf{a} \\
\text { WLW } \\
\text { (toutes } \\
\text { chutes) }\end{array}$ & $\begin{array}{c}\text { 3b } \\
\text { Standard } \\
\text { Cox/WLW } \\
\text { (première } \\
\text { chute) }\end{array}$ & $\begin{array}{c}\mathbf{4 b} \\
\text { AG Cox } \\
\end{array}$ & $\begin{array}{c}\mathbf{5 b} \\
\mathbf{W L W} \\
\\
\text { (toutes } \\
\text { chutes) }\end{array}$ \\
\hline & \multicolumn{5}{|c|}{ avec covariables de base $^{d}$} & \multicolumn{3}{|c|}{$\begin{array}{l}\text { avec covariables } \\
\text { dépendantes du temps }\end{array}$} \\
\hline IMC (kg/m²) & - & - & - & $0,98^{* *}$ & - & - & $0,98^{* *}$ & $0,99^{*}$ \\
\hline Score à l'échelle de Berg & - & $0,98^{* *}$ & - & $0,98^{* * * *}$ & $0,98^{* * *}$ & $0,99^{* *}$ & $0,98^{* * * *}$ & $0,99^{* * * *}$ \\
\hline Score au test Timed Up \& Go & - & - & - & - & $0,99^{* *}$ & - & - & - \\
\hline Homme & $1,47^{*}$ & - & $1,25^{*}$ & - & $1,22^{*}$ & $1,34^{* *}$ & - & $1,30^{* *}$ \\
\hline $\begin{array}{l}\text { Une chute avant l'entrée } \\
\text { dans le projet }{ }^{f}\end{array}$ & $1,95^{* * *}$ & $1,41^{*}$ & $1,47^{* * *}$ & $1,40^{* *}$ & $1,26^{*}$ & $1,45^{* * *}$ & $1,37^{*}$ & $1,24^{*}$ \\
\hline $\begin{array}{l}\text { Deux chutes ou plus avant } \\
\text { l'entréef }^{f}\end{array}$ & $3,28^{* * * *}$ & $3,15^{* * * *}$ & $2,35^{* * * *}$ & $2,31^{* * * *}$ & $2,12^{* * * *}$ & $2,07^{* * * *}$ & $2,21^{* * * *}$ & $1,86^{* * * *}$ \\
\hline
\end{tabular}

Significatif (bilatéral) : ${ }^{*} p \leq 0,05 ;{ }^{* *} p \leq 0,01 ;{ }^{* * *} p \leq 0,001 ;{ }^{* * * *} p \leq 0,0001$.

Durant le suivi, le nombre de chutes antérieures est défini comme une covariable dépendante du temps pour tenir compte du lien de dépendance entre les chutes :

${ }^{\text {a }} \mathrm{RTI}=1,10^{* * * * ;}$ b $\mathrm{RTI}=1,09^{* * * *}$.

' Les sujets ayant fait l'objet d'un suivi pendant moins de 12 mois et n'ayant pas déclaré de chute $(n=221)$ ont été exclus de l'analyse, puisque nous ne pouvions leur reconnaître la condition de personne ayant fait une chute.

d Toutes les covariables à leur valeur initiale seulement; ' les covariables avec une valeur mise à jour comprennent les dangers de l'environnement domiciliaire, I'IMC, le score à l'échelle de Berg et le score au test Timed Up and Go.

${ }^{\mathrm{f}}$ Historique des chutes survenues dans les 3 mois ayant précédé l'entrevue initiale.

On demandait aux participants s'ils avaient fait une ou plusieurs chutes dans les trois mois ayant précédé l'entrevue initiale, et on vérifiait par la suite s'ils avaient fait d'autres chutes par un calendrier à remplir quotidiennement et par une relance téléphonique mensuelle.

On s'est servi d'un indice écologique l'indice de défavorisation matérielle et sociale pour l'étude de l'échantillon; pour ce faire, on a apparié des données du recensement du Canada aux secteurs géographiques de résidence à l'aide des codes postaux ${ }^{27,28}$. De plus, on a évalué le risque nutritionnel au moyen d'un outil de dépistage constitué d'une échelle graduée de 13 points ${ }^{29-32}$. Le participant déclarait son poids corporel, tandis que l'on mesurait la taille du participant par les méthodes usuelles. La démarche, l'équilibre et la mobilité ont été évalués au moyen de l'échelle de Berg ${ }^{33-36}$, constituée de 56 points, et du test " Timed Up and Go $»^{37,38}$, qui sert à mesurer, en secondes, le temps total requis pour accomplir une série de tâches fonctionnelles. On a évalué l'environnement domiciliaire des participants au moyen du formulaire d'évaluation pièce par pièce de Gill ${ }^{39,40}$, en examinant 37 éléments de risque. Les types de logement examinés étaient les suivants : maison unifamiliale; appartement; maison en rangée ou autres unités de logement à entrée unique; résidence privée pour personnes âgées; autres types de logement, dont maison de chambres. Les données sur la consommation de benzodiazépines (oui/non) et la consommation quotidienne de médicaments prescrits (nombre) étaient relevées directement à partir des étiquettes des contenants. Quant à la consommation d'alcool, on pouvait en établir un historique détaillé grâce à un questionnaire mis au point par l'Institut de la statistique du Québec ${ }^{41,42}$. Le répondant devait indiquer s'il avait consommé des boissons alcoolisées au cours de la dernière semaine (oui/non) et s'il avait consommé régulièrement des boissons alcoolisées au cours des 6 derniers mois et à quelle fréquence (ne consomme pas; moins de 4 fois par mois; de 1 à 6 fois par semaine; quotidiennement). En règle générale, une valeur élevée pour ces différents facteurs indiquait un risque accru, sauf dans le cas de l'échelle de Berg, où c'est le contraire.

\section{Analyses statistiques}

Les analyses descriptives ont été effectuées au moyen de $\operatorname{SPSS}^{\circledR} 13.0$ et les analyses de régression, au moyen de $\mathrm{SAS}^{\circledast} 9.1$. Les effets ajustés des caractéristiques des sujets sur la probabilité de chute ont été étudiés à l'aide de trois méthodes d'analyse de survie 
(régression de Cox classique, extension de AG et extension de WLW), de la régression binomiale négative et de la régression logistique ${ }^{15,43}$

Dans toutes les analyses de survie, la variable dépendante est le temps écoulé jusqu'à la chute (en jours), pour chaque participant faisant l'objet du suivi. Seuls les cas pour lesquels on dispose de données sur au moins un mois de suivi sont inclus dans l'analyse. Les sujets sont « censurés » dans l'une ou l'autre des éventualités suivantes : retrait volontaire (facultatif) après un suivi de 18 mois, fin de l'étude, abandon en cours de suivi pour un quelconque motif. Les chutes répétées sont considérées comme les manifestations du même type d'événements indifférenciables. Les analyses de survie ont été effectuées tout d'abord avec les seules covariables de base, puis avec les covariables mises à jour. Les covariables de base comprennent l'âge, le sexe, le nombre de chutes dans les trois mois ayant précédé l'entrée dans le projet, le type de logement et l'indice de défavorisation. Quant aux covariables dépendantes du temps (IMC, risque nutritionnel, consommation d'alcool, dangers de l'environnement domiciliaire, démarche et équilibre, consommation de benzodiazépines et de médicaments divers), on a pris en compte la dernière mesure avant le moment de la chute $^{2}$. Dans le cas des personnes qui n'avaient pas fait de chute, on s'est servi de la mesure de l'exposition au risque au milieu de la période de suivi. Nous avons donc testé l'hypothèse nulle que l'exposition mesurée durant le suivi n'est pas associée au risque de chute ultérieure ${ }^{2}$. L'hypothèse des risques proportionnels était superflue dans la régression de Cox avec covariables dépendantes du temps, puisque les risques étaient liés au temps ${ }^{2,43}$.

La variable dépendante dans la régression logistique est la condition de personne ayant fait une chute (les sujets qui sont tombés au moins une fois) dans une période de
12 mois. La régression binomiale négative et la régression logistique ont été exécutées avec la totalité des covariables de base. La figure 1 résume les méthodes statistiques utilisées. On a vérifié l'hypothèse de la linéarité des relations pour les variables prédictives continues. Tous les modèles ont été ajustés au moyen d'un processus séquentiel visant à conserver le plus grand nombre de variables possibles, étant donné une valeur $p \leq 0,05$. Enfin, on a eu recours à des estimations robustes (« sandwich ») de la variance dans l'analyse de survie et dans la régression binomiale négative, afin de pallier la non-indépendance des événements récurrents, en l'occurrence les chutes.

La méthode de WLW a permis de calculer le $\beta$ commun et le $\beta$ par événement pour les cinq premières chutes de chaque sujet, ainsi que le $\beta$ commun pour l'ensemble des chutes rapportées. Le nombre de sujets exposés au risque pour une strate donnée, après une première chute, comprend tous

TABLEAU 2

Rapport des taux d'incidence selon le modèle de WLW ajusté et corrigé par la variance, pour certains facteurs de risque de chutes chez les aînés vivant dans la communauté, selon le numéro d'ordre de la chute ou la combinaison de chutes

\begin{tabular}{|c|c|c|c|c|c|c|c|}
\hline \multirow[b]{2}{*}{ Facteur de risque } & \multicolumn{5}{|c|}{ Numéro d'ordre de la chute } & \multirow{2}{*}{$\begin{array}{c}5 \text { premières } \\
\text { chutes } \\
n=1843\end{array}$} & \multirow{2}{*}{$\begin{array}{c}\begin{array}{c}\text { Toutes les } \\
\text { chutes }\end{array} \\
n=2169\end{array}$} \\
\hline & $\begin{array}{c}1 \\
n^{\mathrm{a}}=937\end{array}$ & $\begin{array}{c}2 \\
n=429\end{array}$ & $\begin{array}{c}3 \\
n=244\end{array}$ & $\begin{array}{c}4 \\
n=140\end{array}$ & $\begin{array}{c}5 \\
n=93\end{array}$ & & \\
\hline Chutes (nombre) & 442 & 250 & 144 & 99 & 66 & 1001 & 1270 \\
\hline Dangers de l'environ. domicil. (nombre) & $1,12^{* * * *}$ & $1,19^{* * * *}$ & $1,20^{* * * *}$ & $1,17^{* * *}$ & $1,36^{* * * *}$ & $1,16^{* * * *}$ & $1,19^{* * * *}$ \\
\hline IMC (kg/m²) & - & - & $0,95^{* * *}$ & - & - & {$\left[0,99^{*}\right]^{b}$} & $0,99^{*}$ \\
\hline Usage de benzodiazépines & - & $1,37^{*}$ & - & - & - & {$[1,22 * *]$} & {$\left[1,21^{*}\right]$} \\
\hline \multicolumn{8}{|c|}{ Consommation d'alcool au cours des 6 derniers mois } \\
\hline$\leq 2$ fois par mois vs autres catégories & - & - & $1,50^{*}$ & - & - & {$\left[1,20^{*}\right]$} & - \\
\hline Homme & $1,34^{* *}$ & - & - & - & $2,02^{* *}$ & $1,28^{* *}$ & $1,30^{* *}$ \\
\hline Quatrième quartile vs autres quartiles & - & - & - & - & $3,81^{* * * *}$ & - & - \\
\hline Une chute avant l'entrée dans le projet ${ }^{c}$ & $1,45^{* * *}$ & - & - & - & - & $1,37^{* * *}$ & $1,24^{*}$ \\
\hline$\geq 2$ chutes avant l'entrée ${ }^{c}$ & $2,07^{* * * *}$ & $1,65^{* *}$ & $2,15^{* * * *}$ & $1,49^{*}$ & - & $1,95^{* * * *}$ & $1,86^{* * * *}$ \\
\hline
\end{tabular}


les sujets qui ont subi une chute dans la strate précédente, moins ceux qui ont été "perdus » au cours du suivi; le nombre de sujets exposés au risque pour un groupe combiné donné comprend tous les sujets participant à l'étude dans toutes les strates, comme si chacun des sujets représentait dans chaque strate un sujet différent. On a examiné chaque modèle en tenant compte des strates du nombre de chutes antérieures, puis en en faisant abstraction, de sorte que les effets d'autres variables dignes d'intérêt ne soient pas éclipsés ${ }^{6,23}$.

\section{Résultats}

\section{Sujets ayant participé à l'étude}

Des 959 personnes qui répondaient aux critères de participation à l'étude, ont accepté de prêter leur concours et ont reçu la visite d'un professionnel à leur domicile, 22 se sont retirées du projet sans avoir participé à toutes les étapes de l'évaluation ou dans les trente premiers jours de la période de suivi. Pour les 937 autres sujets, la durée moyenne et la durée médiane des périodes de suivi se sont établies à 488 jours et à 458 jours respectivement (intervalle : de 27 à 1330 jours); 549 sujets (soit 57,2\% du total) ont maintenu leur participation durant 12 mois et 377 (39,3\% du total) l'ont poursuivie durant 18 mois. Les répondants étaient en majeure partie des femmes $(75,7 \%)$. L'âge moyen était de 79,5 ans (écart-type de 6,7), et 76,4\% des répondants avaient 75 ans ou plus. Enfin, trente-neuf pour cent (39,0\%) des participants étaient tombés au moins une fois dans les trois mois ayant précédé leur entrée dans le projet, alors que l'événement était survenu plus d'une fois chez 14,9\% des participants.

\section{Comparaison des méthodes statistiques}

Le tableau 1 présente les valeurs du risque relatif de chutes calculées à l'aide de différentes méthodes statistiques. On constate premièrement que la régression logistique (1) et la régression de Cox ordinaire (3a) (avec comme variable dépendante le temps écoulé jusqu'à la première chute) font abstraction de la récurrence des chutes et mettent en évidence un moins grand nombre de facteurs de risque significatifs que la régression binomiale négative (2), la régression de $A G$ (4a) et la méthode de WLW (5a), qui prennent en considération toute l'information disponible (Nota : Le chiffre entre parenthèses renvoie au modèle correspondant dans le tableau 1). Bien que la régression logistique et la régression de Cox ordinaire fassent ressortir les mêmes facteurs de risque, la première ne tient pas compte du moment où survient la chute, ce qui a pour effet de produire des valeurs du risque relatif plus élevées par rapport à la régression de Cox ordinaire. Les valeurs calculées par la régression logistique étaient de 17,6 \% (1,47 contre 1,25$)$ à $39,6 \%(3,28$ contre 2,35$)$ plus élevées que celles calculées par la régression de Cox ordinaire.

Deuxièmement, trois méthodes la régression binomiale négative (2), ainsi que les extensions de AG (4a) et de WLW (5a) du modèle de Cox prennent en compte la durée du suivi, le taux d'incidence global des chutes et le lien de dépendance entre les chutes, grâce, dans ce dernier cas, à des estimations robustes de la variance. La méthode de WLW met en évidence un plus grand nombre de facteurs de risque significatifs que les autres méthodes, mais donne moins d'importance au facteur nombre de chutes survenues dans les trois mois ayant précédé l'entrevue initiale. À ce propos, pour ce qui a trait à la variable deux chutes ou plus avant l'entrée dans le projet, on note un écart de 48,6 \% entre le risque relatif calculé au moyen de la régression binomiale négative $(3,15)$ et celui calculé par la méthode de WLW $(2,12)$. La différence des résultats des trois méthodes s'explique par l'importance que chacune des méthodes accorde au lien de dépendance entre les événements récurrents. La régression binomiale négative fait abstraction de la longueur des intervalles entre les chutes, tandis que le modèle de AG décrit explicitement l'incidence des chutes antérieures sur la probabilité de survenue d'autres chutes. À cet égard, le rapport des taux d'incidence (RTI, qui est l'équivalent du rapport des risques instantanés) de 1,10 rattaché à la variable dépendante du temps nombre de chutes antérieures incluse dans le modèle de AG (4a) signifie que le risque s'accroît de $10 \%$ chaque fois que le nombre de chutes antérieures augmente d'une unité. Par contre, la méthode de WLW calcule des rapports distincts pour chaque chute et calcule les coefficients et la corrélation intra-sujet plus directement que la méthode de AG, ce qui produit des estimations moyennes pondérées efficientes de l'effet (et de la variance).

Troisièmement, nous avons comparé les résultats des modèles qui n'intègrent que les covariables de base et ceux des modèles qui intègrent les covariables dépendantes du temps. Le nombre d'éléments de risque dans l'environnement domiciliaire, facteur particulièrement susceptible de varier durant la période de suivi, n'est pas significativement associé aux chutes de personnes âgées lorsque les modèles considérés ne font intervenir que les covariables de base (1 à 5a). Par contre, la même variable est statistiquement significative lorsqu'on applique les modèles qui font intervenir les covariables dépendantes du temps ( $3 b$ à $5 b$ ). Tous les modèles d'analyse de survie qui intègrent les covariables dépendantes du temps dégagent un plus grand nombre de facteurs de risque que les modèles correspondants qui n'intègrent que les covariables de base (3b vs 3a, $4 \mathrm{~b}$ vs $4 \mathrm{a}$, et $5 \mathrm{~b}$ vs $5 \mathrm{a}$ ), même lorsque les estimations sont calculées en tenant compte d'une variance robuste. On note une différence plus prononcée entre les modèles qui ne considèrent que le temps écoulé jusqu'à la première chute et ceux qui prennent en compte le temps écoulé jusqu'à chaque chute. Lorsque le modèle de WLW fait abstraction des covariables dépendantes du temps, on observe des biais d'orientation variée. Enfin, les méthodes usuelles d'analyse des facteurs de risque de chutes ( $n^{\text {os }} 1$ et 3 dans le tableau 1) produisent des résultats fortement biaisés comparativement à ceux obtenus avec la méthode de WLW qui utilise les covariables dépendantes du temps (5b).

\section{Facteurs de risque de chutes}

L'échantillon formé de 937 sujets a déclaré en tout 1270 chutes durant 457283 personnes-jours d'observation, compte tenu de ce qu'une même personne pouvait déclarer plus d’un événement. 
Sur l'ensemble des sujets, 495 n'ont subi aucune chute, 192 sont tombés une fois et 250 sont tombés plusieurs fois. La prise en compte des cinq premières chutes englobe $90,0 \%$ des 442 personnes qui ont fait une chute et 95,3\% des 937 personnes qui constituent l'échantillon. En ce qui a trait aux conséquences des événements (à tout le moins ceux documentés), précisons que $44,4 \%$ des chutes ont causé des blessures, 25,2 \% ont entraîné une limitation d'activité, 17,1 \% ont mené à une consultation médicale et 5,6\% ont nécessité une hospitalisation. Globalement, $82,1 \%$ des chutes sont survenues dans la maison du participant.

Le tableau 2 présente les rapports d'association ajustés entre les facteurs de risque possibles et le taux d'incidence pour des chutes particulières et une combinaison de chutes. Les valeurs estimées qui figurent dans la première colonne de ce tableau sont en tout point identiques à celles qui sont reproduites dans la colonne $3 \mathrm{~b}$ du tableau 1 , c'est-àdire les estimations que l'on obtient avec un modèle de Cox ordinaire limité aux données sur le temps écoulé jusqu'à la première chute, la seule différence étant que les valeurs $p$ présentées dans le tableau 2 ont été établies sur la base de méthodes statistiques robustes plutôt que sur la base de méthodes standard (" naïves »). S'il est vrai que les estimations relatives à la première chute sont essentiellement les mêmes dans les deux cas précités, les résultats pour les autres strates diffèrent sensiblement selon que les coefficients ont été calculés à l'aide de méthodes robustes ou naïves, ce qui reflète le degré de dépendance entre les événements. Ainsi, le sexe (masculin en l'occurrence), les occupants d'une résidence privée pour personnes âgées, le nombre d'éléments de risque dans l'environnement domiciliaire, le score à l'échelle de Berg et l'âge sont des prédicteurs indépendants et significatifs du temps écoulé jusqu'à la première chute. Par exemple, le RTI de 1,45 calculé pour le facteur résidence privée pour personnes âgées indique que les sujets qui vivent dans un logement de ce type sont tombés $45 \%$ plus souvent que les sujets qui habitent tout autre type de logement. De même, le RTI de 1,12 calculé pour le facteur dangers de l'environnement domiciliaire indique que les risques à domicile augmentent de $12 \%$ chaque fois qu'un élément de risque s'ajoute à la liste. Par contre, comme le RTI pour l'âge est inférieur à 1 (soit 0,98$)$, il faut en déduire que vieillir d'un an entraîne une diminution du risque de $2 \%$.

Le tableau 2 permet aussi de comparer les résultats obtenus lorsqu'on ajuste des $\beta$ distincts pour chaque chute. Des covariables comme l'âge, les dangers de l'environnement domiciliaire et le score à l'échelle de Berg présentent à peu près les mêmes effets d'une strate à l'autre. Dans d'autres cas toutefois, les effets varient selon la nature et l'importance des variables statistiquement significatives, et selon le numéro d'ordre de la chute. Les plus grosses différences dans les RTI s'observent pour la cinquième chute. Enfin, l'inclusion de l'historique des chutes survenues dans les trois mois précédant l'entrée dans le projet donne des résultats hautement significatifs et n'influe pas sur la grandeur ou le caractère significatif du RTI calculé pour les autres variables déjà incluses dans tous les modèles de strate. La section de droite du tableau 2 refait l'analyse suivant les contraintes d'un $\beta$ commun (moyenne pondérée des risques propres à un événement), tout d'abord au moyen des données relatives aux cinq premières chutes (modèle tronqué), puis au moyen des données sur l'ensemble des chutes (modèle intégral). Le modèle tronqué a permis de dégager sept variables, donc trois de plus que le modèle servant à établir le temps écoulé jusqu'à la première chute (à savoir IMC, usage de benzodiazépines, et consommation d'alcool au cours des six derniers mois de suivi) et une de plus que le modèle intégral (consommation d'alcool à l'occasion). Toutefois, ces variables additionnelles ne sont plus significatives, par rapport à la contribution de l'ensemble des autres variables, une fois qu'on a intégré l'historique des chutes dans le modèle tronqué; de plus, l'usage de benzodiazépines et la consommation d'alcool sont non significatifs dans le modèle intégral. Enfin, on a conclu au caractère non significatif d'un terme d'interaction âge-sexe qui a été testé dans chaque modèle final.

\section{Analyse}

Le présent article visait à déterminer la méthode la plus convenable pour l'étude des chutes et de leurs déterminants. Aucune méthode statistique ne peut reproduire parfaitement le comportement humain, et toute tentative de résoudre le problème de l'exposition variable dans le temps et celui des événements récurrents par des solutions improvisées peut mener à de graves erreurs. À notre connaissance, la seule occasion où on a examiné simultanément ces deux problèmes est lors de la rédaction d'une thèse de doctorat qui a été déposée en $1991^{22}$ et publiée ultérieurement dans une revue scientifique $^{23}$. Cela dit, la statistique a fait d'énormes progrès depuis ce temps. Dans le présent numéro, nous avons examiné les diverses méthodes qui servent à étudier la variation de l'exposition au risque durant la période de suivi et la récurrence des événements chez une même personne. Nous avons illustré notre analyse en recherchant les facteurs de risque de chutes chez les aînés. Nous avons concentré notre attention sur les aspects statistiques et méthodologiques et avons formulé des conclusions sur les facteurs de risque dans la mesure où les résultats étaient différents d'une analyse à l'autre.

Il existe maintenant sur le marché des progiciels qui permettent d'exploiter correctement sur le plan statistique les données mentionnées plus haut. Les méthodes contenues dans ces progiciels ont fait l'objet de nombreux ouvrages statistiques, mais elles sont encore peu utilisées dans les études portant sur les chutes, car les progrès réalisés en statistique tardent souvent à se manifester dans le domaine clinique et le domaine de la santé publique ${ }^{4}$. Nous avons exposé dans le présent article les raisons qui nous permettent de croire que la méthode de WLW est un choix judicieux pour les besoins de notre étude. Elle se prête naturellement à l'analyse des données 
sur l'exposition variable dans le temps et la récurrence des événements au moyen d'hypothèses minimales ${ }^{2,44}$. D'autres auteurs signalent en outre la robustesse de cette méthode et sa grande efficacité dans de nombreuses situations concrètes ${ }^{14}$.

Nous avons fait ressortir les différences dans les estimations calculées au moyen de diverses méthodes statistiques d'analyse des facteurs de risque de chutes en fonction de l'information fournie. Les résultats indiquent clairement que les méthodes courantes telles que la régression logistique, où la variable dépendante est binaire, et la régression de Cox ordinaire, qui sert à estimer le temps écoulé jusqu'à la première chute, sont à l'origine de biais considérables, contrairement au modèle de WLW qui utilise des covariables dépendantes du temps. Par ailleurs, lorsqu'on modélise les premiers événements, on suppose implicitement que le premier événement est représentatif de tous les autres. Notre étude indique que cette hypothèse est discutable, et ceci plus dans l'aspect qualitatif des estimations du RTI que dans l'aspect quantitatif. Les résultats de notre étude confirment en outre la pertinence d'utiliser un modèle stratifié plutôt qu'un modèle non stratifié, étant donné que le risque de survenue d'une chute varie considérablement entre les événements. Mahé et Chevret ${ }^{45}$ envisagent cette possibilité lorsque la fréquence des événements par unité est " faible » (p. ex., les chutes chez les aînés vivant dans la communauté).

De plus, les résultats de notre étude concordent avec ceux des études antérieures, sauf que nous sommes plus sûrs de la valeur des estimations des variables prédictives. Il convient ici de commenter quelques-uns de nos résultats. Le nombre d'éléments de risque dans l'environnement domiciliaire et l'historique des chutes sont des prédicteurs importants et stables des chutes de personnes âgées, quels que soient le numéro d'ordre ou le mode de combinaison des chutes. Le fait qu'une personne a déjà subi des chutes par le passé accroît le risque de chutes dans l'avenir, ce qui donne à penser que si on ne supprime pas les causes des chutes antérieures pour lesquelles la variable pertinente sert de substitut, la personne courra plus de risques de subir d'autres chutes causées par les mêmes facteurs ${ }^{23}$. Les personnes qui vivent en résidence privée pour personnes âgées sont plus à risque de chutes que les autres, probablement parce que la variable en question est aussi un indicateur d'état chronique ou d'autonomie réduite. De même, les personnes plus jeunes se révèlent plus à risque de chutes que les personnes plus âgées, probablement parce qu'elles ont une vie plus active.

Nous souhaitons de surcroît écarter tout malentendu concernant les mesures d'incidence décrites dans les ouvrages de recherche, en particulier la mesure discutable événements par personne-temps, qui met le nombre de chutes (unique chez certains sujets, multiples chez d'autres) en rapport avec la durée d'observation cumulée de tous les sujets. Veillons à ne pas confondre cette mesure avec le nombre d'événements, pour un individu, ajusté en fonction de la période de suivi, dont il est question dans le présent article, ou avec le taux d'incidence, couramment utilisé en épidémiologie. Dans le rapport événements par personne-temps, le numérateur ne représente pas le nombre de sujets chez qui l'événement est survenu une seule fois, mais plutôt un nombre d'événements réparti entre les sujets qui participent à l'étude. Windeler et Lange ${ }^{46}$ critiquent vigoureusement ce concept parce qu'on ne peut en donner une interprétation juste au niveau individuel. En conséquence, qu'il s'agisse de 20 sujets observés sur 10 ans et ayant subi chacun deux chutes, ou de 1000 sujets observés sur une demiannée, dont une centaine (10\%) ont subi une seule chute, le résultat est le même (20 pour 100 personnes-années). Introduit dans les années 80 et, malheureusement, faisant encore aujourd'hui l'objet d'articles dans certaines revues à comité de lecture ${ }^{47-50}$, ce concept devrait maintenant être écarté ${ }^{46}$, car il nuit à la recherche de nouvelles approches.

Heureusement, grâce aux études prospectives, aux contacts fréquents, aux mesures répétées et aux évaluations cliniques faites par un thérapeute, on parvient à limiter le biais d'information. Cela dit, les données relatives à certains facteurs de risque, comme le score à l'outil de dépistage nutritionnel et la consommation d'alcool, sont autodéclarées. Il peut se produire des erreurs de classification différentielles si le fait d'une chute ou de chutes récurrentes influe sur la précision avec laquelle les individus se rappellent leur exposition au risque et les conséquences de cette exposition. Ainsi, l'incidence du facteur sur le risque de chute se trouve amplifiée $e^{6}$. Par ailleurs, le temps écoulé entre le moment d'une chute et la mesure de l'exposition au risque durant le suivi varie bien sûr selon le jour où est survenue la chute. Il est donc impossible de déterminer à quel moment exactement, entre chaque période de suivi de six mois, se produit un changement dans l'exposition aux covariables dépendantes du temps, d'où le risque d'erreur non différentielle dans l'évaluation de l'exposition, ce qui affaiblit la relation observée. Une autre source possible de biais est liée aux participants qui abandonnent en cours de route, surtout lorsque ceux-ci ne présentent pas le même taux d'incidence (risque de chute) que les personnesquimaintiennentleurparticipation. Si l'on fait exception des personnes qui ont refusé de recevoir les services et qui couraient un risque moins grand de tomber, par opposition aux participants à l'étude qui ont prêté leur concours jusqu'à la fin, aucun motif de cessation du suivi n'a été associé au risque de chutes. On note que les personnes dont la participation à l'étude est d'une durée beaucoup plus courte que la moyenne sont les hommes, les personnes les plus âgées, les occupants d'une résidence privée pour aînés, les personnes qui appartiennent au premier quartile de l'indice de défavorisation matérielles, celles qui ont obtenu un score moins élevé à l'échelle de Berg et celles qui consomment de l'alcool quotidiennement (valeurs mesurées à l'examen initial). Comme l'ont déjà souligné Campbell et coll. ${ }^{51}$, les personnes plus frêles et possiblement plus à risque de tomber sont également parmi les plus difficiles à recruter et à retenir, ce qui peut se traduire par une sous-estimation des effets.

Toutes ces considérations nous amènent à penser que les résultats de notre étude sont plutôt prudents. Une difficulté que pose sur le plan pratique la méthode de WLW est le fait que l'on doive consacrer au préalable beaucoup d'effort et de soin à la constitution de la base de données. La mise en application de cette méthode repose sur 
le caractère complet des rapports sur les chutes et la connaissance des dates où sont survenus ces événements. Les études futures devront avoir pour objet d'opérer la transposition de l'analyse des facteurs de risque de chutes à la mise en œuvre de mesures d'intervention au sein de la collectivité.

En dernier lieu, il serait utile de commenter deux résultats cliniques importants qui n’ont pas été traités explicitement ici. Premièrement, nous avons estimé le degré auquel le facteur de la démarche et de l'équilibre module la relation entre la consommation de médicaments et la probabilité de chute $^{6}$. Il s'agit ici d'une " variable intermédiaire ", c'est-à-dire d'une variable qui intervient dans la relation causale entre l'exposition et le phénomène étudié. S'il est vrai que cette variable intervient dans la relation causale, l'association entre l'exposition et le phénomène étudié tendra à s'amenuiser lorsqu'on effectuera des ajustements en fonction de la variable intermédiaire ${ }^{52}$. Des ajustements en fonction de l'échelle de Berg se sont traduits par une augmentation maximale de $21 \%$ de l'effet des benzodiazépines, alors qu'on avait supposé qu'il y aurait réduction de cet effet. Par conséquent, la covariable qui nous intéresse ici n'est pas reconnue comme une variable intermédiaire ni comme une variable de confusion notable.

Deuxièmement, nousavonsétudiéleschutes ayant nécessité une consultation médicale comme une autre mesure de résultats, en supposant que cette mesure sert d'indice de gravité de la chute. Nous avons alors défini comme covariable dépendante du temps la variable " chutes antérieures ", cela dans le but d'examiner si une chute n’ayant pas nécessité une consultation médicale avait été déclarée dans les trois mois précédant une consultation médicale liée à une chute. Les valeurs ajustées ont permis de déterminer que les dangers de l'environnement domiciliaire (rapport des taux d'incidence $=1,09$ ), le score à l'outil de dépistage nutritionnel $(1,09)$, le fait de vivre dans une résidence privée pour aînés $(1,67)$ et l'historique des chutes (une chute avant la chute considérée =
0,59; deux chutes ou plus avant la chute considérée $=0,64$ ) étaient des prédicteurs significatifs et indépendants des consultations médicales liées aux chutes dans leur ensemble. Le fait d'avoir fait une chute dans les trois mois précédant chaque nouvel événement étudié était un facteur de protection contre toute chute nécessitant une consultation médicale.

\section{Remerciements}

Les auteurs tiennent à remercier tous les aînés et les professionnels des centres de santé et de services sociaux de Lanaudière qui ont participé à l'étude. Ils expriment également leur gratitude envers Josée Payette pour la collaboration spéciale qu'elle leur a fournie en préparant les fichiers de données qui ont servi aux analyses, ainsi que Nancy Leblanc, Julie Meloche et Jean-François Allaire, du Centre de recherche de l'Institut Philippe-Pinel de Montréal, pour les calculs statistiques des analyses de régression, et Bruce Charles Bezeau, pour la révision du manuscrit en anglais. La recherche a été commanditée par l'Agence de la santé et des services sociaux de Lanaudière.

\section{Références}

1. Ministère de la Santé et des Services sociaux du Québec. La prévention des chutes dans un continuum de services pour les aînés vivant à domicile : Cadre de référence, Québec, Direction générale de la santé publique, 2004, $61 \mathrm{p}$.

2. Desquilbet L, Meyer L. Variables dépendantes du temps dans le modèle de Cox. Théorie et pratique, Rev Épidemiol Santé Publique 2005;53(1):51-68.

3. Moulton LH, Dibley MJ. Multivariate timeto-event models for studies of recurrent childhood diseases. Int $\mathrm{J}$ Epidemiol 1997;26(6):1334-9.

4. Mahé C, Chevret S. Estimation of the treatment effect in a clinical trial when recurrent events define the endpoint. Stat Med 1999;18(14):1821-9.
5. Glynn RJ, Buring JE. Counting recurrent events in cancer research. J Natl Cancer Inst 2001;93(7):488-9.

6. Cumming RG, Kelsey JL, Nevitt MC. Methodologic issues in the study of frequent and recurrent health problems. Falls in the elderly. Ann Epidemiol 1990;1(1):49-56.

7. Goodman AC, Hankin JR, Kalist DE, Peng Y, Spurr SJ. Estimating determinants of multiple treatment episodes for substance abusers. J Ment Health Policy Econ 2001;4(2):65-77

8. Robertson MC, Campbell AJ, Herbison P. Statistical analysis of efficacy in falls prevention trials. J Gerontol A Biol Sci Med Sci 2005;60(4):530-4.

9. Wang SJ, Winchell CJ, McCormick CG, Nevius SE, O'Neill RT. Short of complete abstinence: an analysis exploration of multiple drinking episodes in alcoholism treatment trials. Alcohol Clin Exp Res 2002;26(12):1803-9.

10. Hogan DB, MacDonald FA, Betts J, Bricker S, Ebly EM, Delarue B, Fung TS, Harbidge C, Hunter M, Maxwell CJ, Metcalf B. A randomized controlled trial of a community-based consultation service to prevent falls. CMAJ 2001;165(5):537-43.

11. Glynn RJ, Buring JE. Ways of measuring rates of recurrent events. BMJ 1996; 312(7027):364-7.

12. Lin DY. Cox regression analysis of multivariate failure time data: the marginal approach. Stat Med 1994;13(21):2233-47.

13. Finkelstein DM, Schoenfeld DA, Stamenovic E. Analysis of multiple failure time data from an AIDS clinical trial. Stat Med 1997;16(8):951-61.

14. Wei LJ, Glidden DV. An overview of statistical methods for multiple failure time data in clinical trials. Stat Med 1997;16(8):833-9. 
15. Cleves M. How do I analyze multiple failure-time data using Stata? Stata FAQ, 2002, 11 p., URL : http://www.stata.com/ support/faqs/stat/stmfail.html, date de consultation : 14 juillet 2007.

16. Andersen PK, Gill DR. Cox's regression model for counting processes. Ann Statist 1982;10(4):1100-1120.

17. Prentice RL, Williams BJ, Peterson $\mathrm{AV}$. On the regression analysis of multivariate failure time data. Biometrika 1981;68(2):373-379.

18. Wei LJ, Lin DY, Weissfeld L. Regression analysis of multivariate incomplete failure time data by modelling marginal distributions. J Am Stat Assoc 1989; 84(408):1065-73.

19. Lancar R. Méthodes robustes d'analyse de temps de survie multivariés, Rev Épidemiol SantÉ Publique 1999;47(3):287-96.

20. Robertson, MC. Development of a falls prevention programme for elderly people: Evaluation of efficacy, effectiveness, and efficiency, thèse de doctorat, University of Otago, Department of Medical and Surgical Sciences, Dunedin (Nouvelle-Zélande), 2001.

21. D'Agostino RB, Lee ML, Belanger AJ, Cupples LA, Anderson K, Kannel WB. Relation of pooled logistic regression to time dependent Cox regression analysis: the Framingham Heart Study. Stat Med 1990;9(12):1501-15.

22. O'Loughlin J. The incidence of and risk factors for falls and fall-related injury among elderly persons living in the community, thèse de doctorat, Université McGill, Département d'épidémiologie et de biostatistique, Montréal (Québec), Canada, 1991, 270 p.

23. O'Loughlin JL, Robitaille Y, Boivin JF, Suissa S. Incidence of and risk factors for falls and injurious falls among the community-dwelling elderly. Am J Epidemiol 1993;137(3):342-54.
24. Nevitt MC, Cummings SR, Kidd S, Black D. Risk factors for recurrent nonsyncopal falls. A prospective study. JAMA 1989;261 (18):2663-8.

25. Fletcher PC, Hirdes JP. Risk factors for falling among community-based seniors using home care services. J Gerontol A Biol Sci Med Sci 2002;57(8):M504-10.

26. Bégin $\mathrm{C}$. Projet-pilote régional de prévention des chutes à domicile chez les personnes âgées : Devis d'implantation dans les CLSC, Saint-Charles-Borromée, Service de prévention et de promotion, Direction de santé publique, Régie régionale de la santé et des services sociaux de Lanaudière, 2002, 120 p.

27. Pampalon R, Hamel D, Raymond G. Indice de défavorisation pour l'étude de la santé et du bien-être au Québec - Mise à jour 2001, Institut national de santé publique du Québec, 2004, 11 p., URL : http:// www.inspq.qc.ca/pdf/publications/295IndiceDefavorisation_2001.pdf, date de consultation : 24 décembre 2006.

28. Leclerc BS, Marquis G, Payette J. Tableau de bord lanaudois sur la défavorisation. Territoire de Lanaudière. Calibrage à l'échelle de la région de Lanaudière, Joliette, Agence de la santé et des services sociaux de Lanaudière, Direction de santé publique et d'évaluation, Service de surveillance, recherche et évaluation, 2005, $87 \mathrm{p}$.

29. Payette H. Développement, validation et évaluation d'un programme de dépistage nutritionnel pour les personnes âgées en perte d'autonomie vivant dans la communauté, Sherbrooke, Centre de recherche en gérontologie et gériatrie, Centre d'expertise en gérontologie et gériatrie inc., Institut universitaire de gériatrie de Sherbrooke, non daté, pages multiples.

30. Payette H, Guigoz Y, Vellas BJ. Study design for nutritional assessments in the elderly ", in Methods in Aging Research, B.P. YU (ed), Boca Raton (Florida), CRC Press LLC, 1999, p. 301-20.
31. Laporte M, Villalon L, Payette H. Simple nutrition screening tools for healthcare facilities: development and validity assessment. Can J Diet Pract Res 2001; 62(1):26-34.

32. Laporte M, Villalon L, Thibodeau J, Payette H. Validity and reliability of simple nutrition screening tools adapted to the elderly population in healthcare facilities. J Nutr Health Aging 2001;5(4):292-4.

33. Berg K. Balance and its measure in the elderly: a review. Physiother Can 1989;41(5):240-6.

34. Berg KO, Maki BE, Williams JI, Holliday PJ, Wood-Dauphinee SL. Clinical and laboratory measures of postural balance in an elderly population. Arch Phys Med Rehabil 1992;73(11):1073-80.

35. Berg KO, Wood-Dauphinee SL, Williams JI, Maki B. Measuring balance in the elderly: validation of an instrument. Can J Public Health 1992;83 (suppl. 2):S7-11.

36. Berg K, Wood-Dauphinee S, Williams JI. The Balance Scale: reliability assessment with elderly residents and patients with an acute stroke. Scand J Rehabil Med 1995;27(1):27-36.

37. Podsiadlo D, Richardson S. The timed «Up \& Go»: a test of basic functional mobility for frail elderly persons. J Am Geriatr Soc 1991;39(2):142-8.

38. Lin MR, Hwang $\mathrm{HF}$, $\mathrm{Hu} \mathrm{MH}$, Wu HD, Wang YW, Huang FC. Psychometric comparisons of the timed up and go, one-leg stand, functional reach, and Tinetti balance measures in communitydwelling older people. J Am Geriatr Soc 2004;52(8):1343-8.

39. Gill TM, Williams CS, Robison JT, Tinetti ME. A population-based study of environmental hazards in the homes of older persons. Am J Public Health 1999;89(4):553-6. 
40. Gill TM, Williams CS, Tinetti ME. Environmental hazards and the risk of nonsyncopal falls in the homes of community-living older persons. Med Care 2000;38(12):1174-83.

41. Chevalier S, Lemoine O. Consommation d'alcool, in Enquête sociale et de santé 1998, Québec, Institut de la statistique du Québec, 2000, p. 117-33. (Collection La santé et le bien-être)

42. Institut de la statistique du Québec Annexe 3. Questionnaire autoadministré (QAA) VI - L'alcool, dans Enquête sociale et de santé 1998, Collection La santé et le bienêtre, Québec, Institut de la statistique du Québec, 2000, p. 15-17.

43. Allison PD. Survival analysis using SAS: A practical guide. Cary NC: SAS Institute inc., 1995, 304 p.
44. Li QH, Lagakos SW.Use of the Wei-LinWeissfeld method for the analysis of a recurring and a terminating event. Stat Med 1997;16(8):925-40.

45. Mahé C, Chevret S. Analysis of recurrent failure times data: should the baseline hazard be stratified? Stat Med 2001; 20(24):3807-15.

46. Windeler J, Lange S. Events per person year - a dubious concept. BMJ 1995; 310(6977):454-6.

47. Saari P, Heikkinen E, Sakari-Rantala R, Rantanen T. Fall-related injuries among initially 75- and 80-year old people during a 10-year follow-up. Arch Gerontol Geriatr 2007;45(2):207-15.

48. Sambrook PN, Cameron ID, Chen JS, Cumming RG, Lord SR, March LM, Schwarz J, Seibel MJ, Simpson JM. Influence of fall related factors and bone strength on fracture risk in the frail elderly. Osteoporos Int 2007;18(5):603-10.
49. Cook WL, Tomlinson G, Donaldson M, Markowitz SN, Naglie G, Sobolev B, Jassal SV. Falls and fall-related injuries in older dialysis patients. Clin J Am Soc Nephrol 2006;1(6):1197-204.

50. Mahoney JE, Palta M, Johnson J, Jalaluddin M, Gray S, Park S, Sager M. Temporal association between hospitalization and rate of falls after discharge. Arch Intern Med 2000;160(18):2788-95.

51. Campbell AJ, Robertson MC, Gardner MM, Norton RN, Buchner DM. Falls prevention over 2 years: a randomized controlled trial in women 80 years and older. Age Ageing 1999;28(6):513-8.

52. Szklo M, Nieto FJ. Epidemiology: Beyond the basics, Gaithersburg (MD), Aspen Publications, 2000, 494 p. 